\title{
Design and Implementation of 3D Resources Management System for SMMEs Based on Ajax and Web3D
}

\author{
Ming $\mathrm{Zu}$, Tiemeng Li, Xiaowei Liu and Wenjun Hou \\ Automation School, Beijing University of Posts \& Telecommunications, Beijing 100876, \\ P.R. China diysimon@gmail.com tiemeng2000@gmail.com liuxiaowei1983@me.buaa.edu.cn \\ wenjunh2113@263.net
}

\begin{abstract}
Three-dimensional (3D) files are very important resources for Small \& Middle-sized Manufacturing Enterprises (SMMEs). In this paper we present a system for management and exhibition of product in 3D form. Virtual Reality Modeling Language (VRML) and extension 3D (X3D) in our system are used to achieve the visualization of model on the internet. Users could view these models only through a web browser. Asynchronous JavaScript and XML (Ajax) also are used in our system to enhance the user experience. Web Services is also introduced in our system. Users only need to access some services that they want to build a virtual exhibition platform without lots of development work. A simple prototype will be introduced in the end.
\end{abstract}

Keywords: Ajax, Manufacturing, Virtual reality, Web Services, X3D

\section{INTRODUCTION}

In the process of the SMMEs' operation, 3D model files play two important roles. One is 3D model files used in the system such as CAD, CAM and CAPP are very important file resource for improving the automation degree of the enterprise. On the other hand, 3D model files can be used in virtual display, and this is an effective, intuitionistic method for product publicity.

Based on these requirements mentioned above, we present a platform built with Web Services. On this platform users can conveniently build a distributed system as an effective management system for the 3D files or a $3 \mathrm{D}$ virtual exhibition environment through the services which they need.

In this system, X3D is used to describe and storage the 3D models. We assume that Browser - Server structure $(\mathrm{B} / \mathrm{S})$ will be adopted when they build themselves' system. We use Ajax which is a powerful technology for building rich internet application to exchange the data and enhance the interaction.

In this paper, we present the concept and architecture of the system, and discuss some important work and key points.

Please use the following format when citing this chapter:

Zu, M., Li, T., Liu, X., Ilou, W.. 2007, in IIIP International Federation for Information Processing. Volume 254. Research and Practical Issues of Finterprise Information Systems II Volume 1, eds. I. Xu, Tjoa A.. Chaudhry S. (Boston: Springer), pp. 529-533. 


\section{USING X3D AND AJAX}

There are many methods to realize the 3D sense viewing in the browser. Currently, the most popular technology is VRML/X3D, after installed VRML/X3D plug-in on the client, user can view the 3D scene and do some simple interaction with the model in the browser directly with a VRML/X3D plug-in, e.g. BS Contact VRML, Cortona VRML Client.

Using VRML/X3D to realize the display in the browser of the 3D products refers to the follow key points: the linkages between different products; the interaction between the products and sense; the interaction between the sense and users; the interaction between different users.

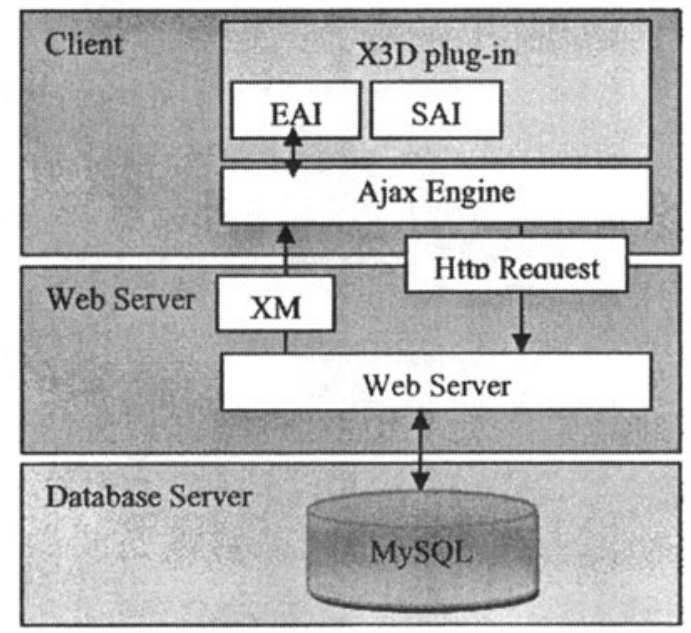

Figure 1. System Architecture

VRML/X3D will not help us to solve all the problems, but X3D provide SAI (Scene Application Interface) and EAI (External Application Interface) that make the VRML/X3D can exchange data with external script language and applications. Usually people use JAVA as an external language to communicate with VRML/X3D directly. But in this way, the efficiency and interaction are generally not excellent.

So we use Ajax to be a middleware. We transmit the XML data through JavaScript running on client. It just refreshes some part of web page which is modified. This method breaks the traditional practice of reload pages. Using Ajax, Web pages can be partially reloaded directly without interruption to the interaction flows. This makes it possible that user can build a Web application close to the local desktop application.

This makes the data' communication and control from sever to 3D sense come true, so we will be able to achieve many functions in $3 \mathrm{D}$ environment. 


\section{USING WEB SERVICES}

The feeling of immersion is one of key experience in the virtual reality environment. In a virtual reality environment we not only want to examine a product itself, sometimes the environment which the product is in is necessary that it could help users understand the product. The environment can enhance the authenticity and immersion, this can make users feel they are there, and get a good display result.

But we have to face a fact that if each enterprise has to build different environment for their different products, which make the cost high. Moreover, building 3D model and $\mathrm{X} 3 \mathrm{D}$ programming are not very easy for every developer.

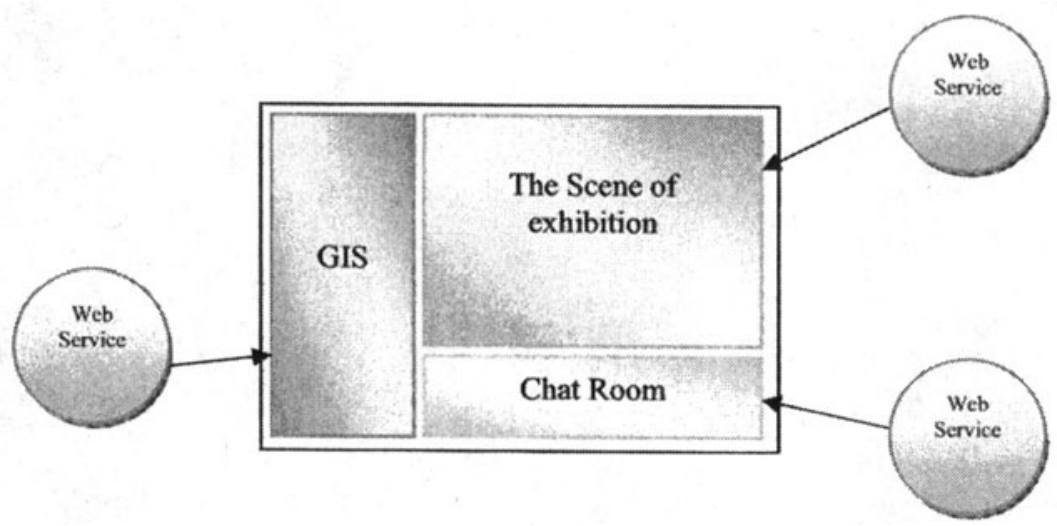

Figure 2. A Prototype Using Web Services

Trough the Web Services, we can pack different environment and scene into several services, and provide them to users who want to build virtual platform. That makes having a products virtual display much easier for developers. Obviously it will encourage users to publish their products in this way. e.g. we build a gallery scene for electrical exhibition, then if an electrical manufacture wish to have an virtual products display the only thing he need to do is using our gallery scene and put his $3 \mathrm{D}$ model files of products in.

Besides the scene, we can add a lot of subsidiary functional modules as many web services such as in the part of Chat Room, Product News, etc, the services from different places will be put into one platform, which just likes the Google Personalized Home. This will greatly reduce the development costs and improve efficiency. 


\section{PROTOTYPE DESIGN}

\subsection{Interaction of 3D models}

The most of X3D plug-in support the zooming, rotation, displacement of models. These actions are all view action. In addition, we also need some acts that are active control behavior, the most important thing is selecting object.

X3D provides a series of event interface, which include mouse click event. When user clicks one model, X3D will trigger the event "eventOut_mouseDown". Then it will map to a corresponding function in JavaScript program. At the same time, the information of node in model will be transmitted to JavaScript. JavaScript procedures for handling such information or transmits to the server. When a result is returned from server, JavaScript will announce the X3D to modify the information of model. This is a flow of interaction in system.

\subsection{Navigation}

- During viewing the virtual scene, every model is a part of the virtual scene. When the space of virtual scene is very big, visitor needs a navigation helping him to find the information he want. Basic on different needs we design two kinds of navigations for visitors.

Two-dimensional map navigation: in a big virtual scene, the visitor's experience of viewing in it is just like walking in a real environment, so it is helpful to be provided a two-dimensional map. We set a background clock, and every clock cycle X3D will tells JavaScript program the location of visitor. And then JavaScript program will judge the distance between the location of this clock cycle and last one, if the distance is long enough to show on the two-dimensional map, JavaScript will conversion the visitor' location information in order to complete the two-dimensional map updates.

Three-dimensional navigation: sometimes user need to view some part of object by select it intuitively. We supply a 3D view of the whole object. System could response to click from users and catch the part which is selected by user. Then show it in the main view.

\subsection{Web Services}

We divide web services into two categories, one is about 3D, and the other is not.

The services about 3D are scene supply. This type of services needs the VRML model and X3D file of scene. When the system accesses the scene services, the scene will be loaded in the system.

The services unrelated to 3D mostly include message boards, chat rooms, news and a series of traditional web application. 

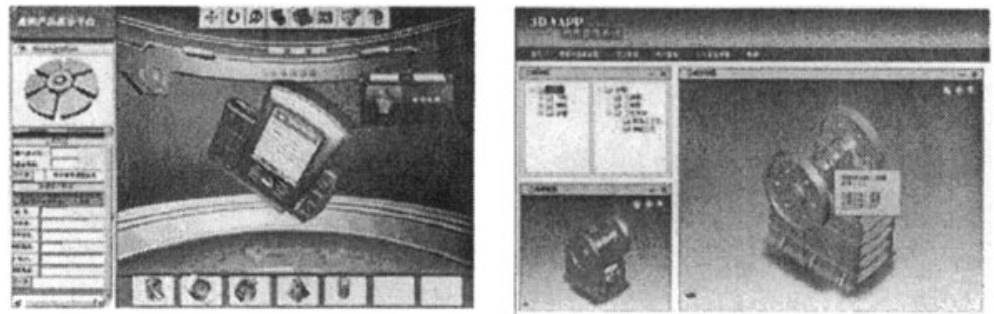

Figure 3. Prototype

\section{CONCLUSIONS}

In this paper, we have presented the concept of 3D virtual reality environment for management and exhibition the 3D models of SMMEs' products. We mainly introduce the transmission of 3D data between the server and client and building the distributed platform using Web Services. We can implement a 3D rich internet application with X3D and Ajax. These two technologies not only solve the communication problem but also improve the interaction of 3D scene. The development cost of enterprise could be reduced because Web Services enhance the reusability of function model.

In the future, we will study the multi-user collaboration problem. In particular people how to work together in $3 \mathrm{D}$ virtual reality environment.

\section{REFERENCES}

1. D. Crane and E. Pascarello, Ajax in Action (2006). http://www.turingbook.com

2. J.J. Garrett. Ajax: A New Approach to Web Applications, Adaptive Path (Feb. 2005).

3. L.D. Paulson, Building rich web applications with Ajax, Computer, IEEE. Volume 38, Number 10, pp.14-17, (2005).

4. X. Tian, C. Huang, and H. Chen, Cache Hint Generation Based on a Profile of an OPT Cache Replacement, Computer Engineering. Volume 31, pp.85-87, (2005).

5. W. Hou, Y. Yan, W. Duan, and H. Shun, Research On Three Dimensional Computer Assistance Assembly Process Design System, Journal of Wuhan University of Technology. Volume 28, Number 83, pp.1088-1092, (2006). 